\title{
Modeling of Fatigue Crack Propagation in Aluminum Alloys Using an Energy Based Approach
}

\author{
F. Khelil \\ Laboratoire de Mécanique \\ de Lille (LML), University \\ of Lille1, France \\ foudil.khelil@univ-lille1.fr
}

\author{
B. Aour \\ Laboratory of Environmental \\ Technology Research, ENSET \\ d'Oran, Algeria \\ Ben_aour@yahoo.fr
}

\author{
M. Belhouari \\ Department of Mechanical \\ Engineering, University of Sidi \\ Bel Abbes, Algeria \\ m_belhouari@yahoo.com
}

\author{
N. Benseddiq \\ LML, University of Lille1, \\ France \\ noureddine.benseddiq@ \\ univ-lille1.fr
}

\begin{abstract}
Materials fatigue is a particularly serious and unsafe kind of material destruction. Investigations of the fatigue crack growth rate and fatigue life constitute very important and complex problems in mechanics. The understanding of the cracking mechanisms, taking into account various factors such as the load pattern, the strain rate, the stress ratio, etc., is of a first need. In this work an energy approach of the Fatigue Crack Growth (FCG) was proposed. This approach is based on the numerical determination of the plastic zone by introducing a novel form of plastic radius. The experimental results conducted on two aluminum alloys of types 2024-T351 and 7075-T7351 were exploited to validate the developed numerical model. A good agreement has been found between the two types of results.
\end{abstract}

Keywords- fatigue crack growth; energetic approach; Plastic zone; aluminum alloys.

\section{INTRODUCTION}

In these last years, the concepts of fracture mechanics allowed a better definition of the stresses and strains fields at the vicinity of crack tips under static and dynamic loadings. Cracking laws, empirical or formal, were developed in order to explore with an acceptable approximation the fatigue crack growth. Indeed, cracks' growth is related to the existence of a plastic zone (PZ) at the crack tip, the formation and the intensification of which is accompanied by energy dissipation. Thus, the amount of the cyclic plastic strain energy may represent with precision the rate of damage at the crack tip.

The use of a cyclic plastic dissipation criterion for fatigue crack growth was first proposed by Rice [1]. From this date, plastic energy approaches to fatigue crack extension prediction have been the subject of several experimental, analytical and numerical investigations [2-10]. Weertman [11] proposed that the crack advances when the accumulated plastic energy at the crack tip reaches a critical value. Then, Shozo et al [2] measured the cyclic work to produce a unit area of fatigue crack for a steel of low carbon content and for high resistance aluminum alloys, using micro strain gages stuck in the plastic zone associated with a fatigue crack. Subsequently, different techniques have been developed to evaluate the plastic energy, such as sub-grain size measurements [3], infrared thermography [4], micro-calorimetry [6] and by direct measurement of hysteresis energy under the loading line of a compact tensile (CT) specimen [12, 20].
Following the work of Bodner et al [5], Klingbeil [7] has proposed a crack growth law, in which the fatigue crack growth rate was related to the total plastic energy dissipated ahead of a crack tip under cyclic loading. This model has been further extended to mixed mode fatigue delamination of layered materials across the interface [10, 14]. Recently, Mazari et al [15] proposed an empirical correction factor which takes into account the over evaluations obtained by hysteresis loops and shows the different effects of plasticity, crack closure and opening mode.

In this paper, a new approach, for the evaluation of the cyclic plastic strain energy at the crack tip in mode I, has been proposed. This approach is based on the numerical determination of the plastic zone by introducing a novel form of plastic radius. The theoretical basis related to the surface energy creation and the evolution of the energy parameters will be discussed in section 2. Section 3 is devoted to the presentation of the experimental data exploited for the validation. Then, section 4 describes the development of the numerical algorithm used for the evaluation of the cyclic plastic strain energy. The obtained results are presented and discussed in the last section.

\section{THEORETICAL BACKGROUND}

\section{A. Energetic description of fatigue crack growth}

The description of kinetics of fatigue failure is very important for estimating fatigue lifetime of a component. The knowledge of crack propagation direction and crack growth rate makes it possible to predict the lifetime by means of Kinetics Fatigue Failure Diagrams (KFFD). However, for an estimation of the cyclic plastic strain energy $\Delta W p$, the area of the hysteresis loop (Figure 1), which characterizes the energy corresponding to one loading cycle, can be used. To this end, a power law relationship between stress and strain has been proposed by Morrow [13] as follows:

$$
\Delta W=\frac{1-N^{\prime \prime}}{1+N^{\prime \prime}} \Delta \sigma \cdot \Delta \varepsilon
$$

where $N^{\prime \prime}$ is the exponent linking the stress amplitude $\Delta \sigma$ and the plastic strain amplitude $\Delta \varepsilon_{p}$. 
On the other hand, a specific energy, $U$, is defined as,

$$
U=\frac{\Delta W_{p}}{2 B \times d a / d N}
$$

where $B$ is the specimen thickness and $d a / d N$ is the fatigue crack growth rate.

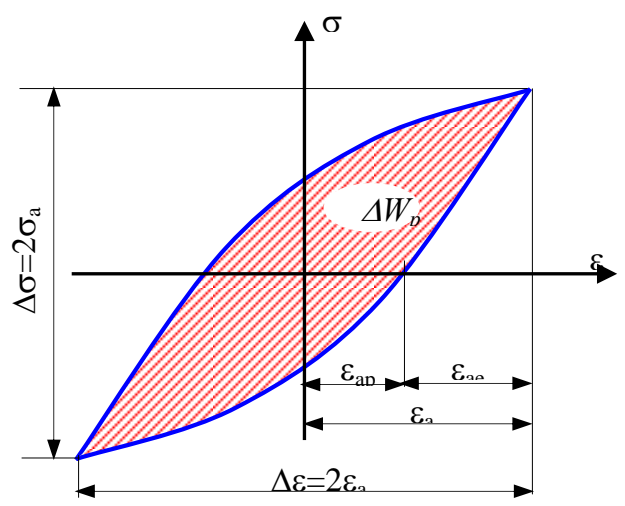

Fig. 1. Schematic drawing showing hysteresis loop.

The evolution of $U$ as a function of $d a / d N$ can be subdivided according to three stages of the KFFD. It should be noted that this diagram can be easily obtained experimentally by measuring crack propagation as a function of the stress intensity factor (see Figure 2). The obtained curve is characterized by three stages which are commonly referred to as stage I, II and III respectively [16, 17].

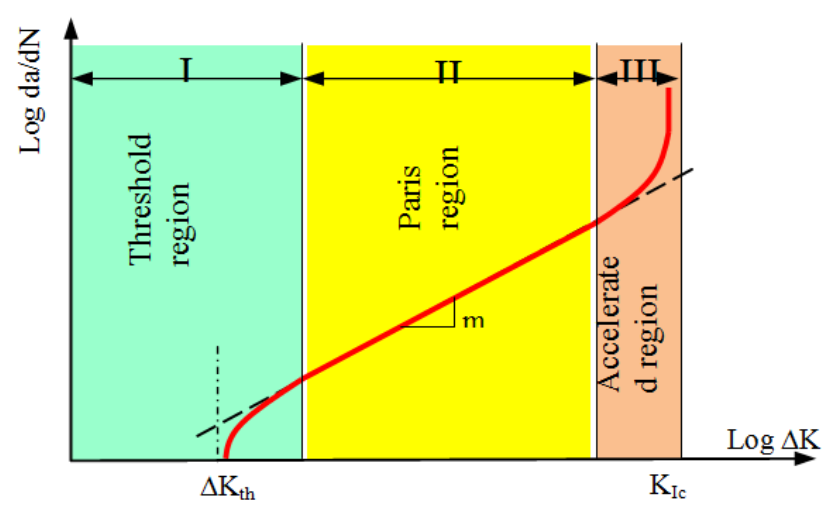

Fig. 2. A typical fatigue crack growth rate curve (kinetic fatigue failure diagram: KFFD).

The relation between $R$ and $\log \Delta K$ is linear [11, 18, 19], whereas that between $d a / d N$ and $U$ can be written as:

$$
\frac{d a}{d N}=\frac{A \Delta K^{4}}{G \sigma_{c}^{2} U}
$$

\section{B. The cyclic plastic strain enery-Proposed model}

Assuming that the energy is primarily dissipated in the plastic zone, a comparison can be made between the measured values and those predicted theoretically by assuming propagation in mode I. Rice [1] and Tracey [21] gave an expression for the equivalent shear strain $\bar{\gamma}=\bar{\varepsilon} \sqrt{3}$ near the crack tip defined in terms of the amplitude function $R_{N}(\theta)$ as follows:

$$
\bar{\gamma}=\tau_{0} / G\left(R_{N}(\theta) / r\right)^{\frac{1}{1+N^{\prime}}}
$$

where $r$ and $\theta$ are the polar coordinates at the crack tip, $G$ the shear modulus, $\tau_{0}=\sigma_{0} / \sqrt{3}$ the yield stress under pure shear and $R_{N}(\theta)$ can be considered the dominant singular term approximation to the elastic-plastic boundary which depends upon the hardening exponent $N^{\prime}$ and is given as a function of $\theta$ in the normalized form [21]:

$$
f_{N}(\theta)=R_{N}(\theta) /\left(K / \sigma_{0}\right)^{2}
$$

where $f_{N}(\theta)$ is a dimensionless function which defines the profile of iso-deformation as a function of polar coordinates at the crack tip.

According to Rice [1], the singularity of equivalent cyclic strain can be described by applying the tensile form of (4-5) by simply replacing $K$ by $\Delta K$ and $\sigma_{0}$ by $\Delta \sigma_{0}$ related to the cyclic stress-strain law.

$$
\overline{\Delta \varepsilon}=\Delta \varepsilon_{p}+\overline{\Delta \sigma} / 3 G
$$

where $\Delta \varepsilon_{p}$ and $\overline{\Delta \sigma}$ are the plastic strain and stress amplitude. Consequently the cyclic stress strain curve gives the hardening law for the material at the crack tip [22]:

$$
\frac{\overline{\Delta \sigma}}{\Delta \sigma_{0}}=\left(\frac{\overline{\Delta \varepsilon}}{\Delta \varepsilon_{0}}\right)^{N^{\prime}}
$$

where $\Delta \varepsilon$ and $\overline{\Delta \sigma}$ are the equivalent strain and stress amplitude, $\Delta \sigma_{0}$ and $\Delta \varepsilon_{0}=\Delta \sigma_{0} / 3 G$ represent the cyclic yield strength of the material.

Using the expressions $\overline{\Delta \gamma}=\overline{\Delta \varepsilon} \sqrt{3}$ and $\tau_{0}=\sigma_{0} / \sqrt{3}$, equation (4) can be rewritten as follows :

$$
\overline{\Delta \varepsilon}=\frac{\Delta \sigma_{0}}{3 G}\left(\frac{R_{N}(\theta)}{r}\right)^{1 / 1+N^{\prime}}
$$

Therefore within the hypothesis given by Rice [1], the equivalent strain amplitude near the crack tip is given by:

$$
\overline{\Delta \varepsilon}=\Delta \varepsilon_{0}\left[\frac{f_{N}(\theta)}{r} \cdot\left(\frac{\Delta K}{\Delta \sigma_{0}}\right)^{2}\right]^{1 / 1+N^{\prime}}
$$


where $\Delta K$ is the stress intensity range which can be given as a function of the maximum and minimum stresses and the crack length a as follows: $\Delta K=\left(\sigma_{\max }-\sigma_{\min }\right) \sqrt{\pi a}$.

In order to evaluate the amplitude of the equivalent average strain $\langle\overline{\Delta \varepsilon}\rangle$ Chalant [23] considered an element with a rectangular or circular form located at the crack tip so that:

$$
\langle\overline{\Delta \varepsilon}\rangle=\frac{1}{S} \int_{0}^{S} \Delta \varepsilon d S
$$

where $S$ is the surface of the element at the crack tip.

In the case of a rectangular element with dimensions $D_{1}$ and $D_{2}$ (see Figure 3a), we get $[16,23]$ :

$$
\langle\overline{\Delta \varepsilon}\rangle=\frac{1+N^{\prime}}{1+2 N^{\prime}} \Delta \varepsilon_{0}\left(\frac{\Delta K}{\Delta \sigma_{0}}\right)^{\frac{2}{1+N^{\prime}}}\left(D_{1}^{\frac{-1}{1+N^{\prime}}} I_{1}+D_{2}^{\frac{-1}{1+N^{\prime}}} I_{2}\right)
$$

The terms $I_{1}$ and $I_{2}$ are given by:

$$
\begin{aligned}
& I_{1}=\int_{0}^{\theta_{0}}\left[\frac{f_{N}(\theta)}{(\cos \theta)^{1+2 N^{\prime}}}\right]^{\frac{1}{1+N^{\prime}}} \cdot d \theta \\
& I_{2}=\int_{\theta_{0}}^{\pi / 2}\left[\frac{f_{N}(\theta)}{(2 \sin \theta)^{1+2 N^{\prime}}}\right]^{\frac{1}{1+N^{\prime}}} \cdot d \theta
\end{aligned}
$$

with

$$
\theta_{0}=\tan ^{-1} \frac{D_{2}}{2 D_{1}}
$$

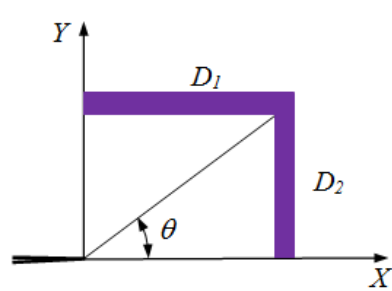

(a)

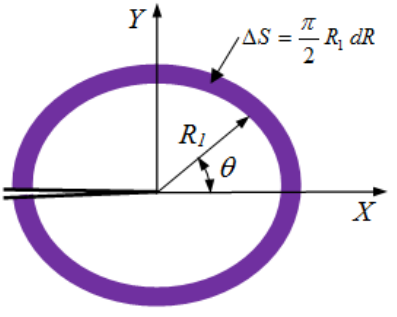

(b)
Fig. 3. Elements at crack tip: (a) rectangular, (b) circular. The drawing shows the hysteresis loop.

In the case of a circular crack tip element with a radius $R_{1}$ (Figure $3 \mathrm{~b}$ ), the expression of the amplitude of the equivalent average strain is given by [12]:

$$
\langle\overline{\Delta \varepsilon}\rangle=\Delta \varepsilon_{0}\left(\frac{\Delta K}{\Delta \sigma_{0}}\right)^{\frac{2}{1+N^{\prime}}}\left(R_{1}^{\frac{-1}{1+N^{\prime}}}-\frac{2}{\pi} I_{\theta}\right)
$$

with

$$
I_{\theta}=\int_{0}^{\pi / 2}\left[f_{N}(\theta)\right]^{\frac{1}{1+N^{\prime}}} d \theta
$$

The plastic energy throughout the plastic zone is obtained by integrating the plastic energy per surface element given by (1), i.e.

$$
\Delta W(P Z)=4 \frac{1-N^{\prime \prime} S_{p}}{1+N^{\prime \prime}} \bar{\Delta}^{0} \overline{\Delta \sigma} \cdot \overline{\Delta \varepsilon}_{p} \cdot d S
$$

where $S_{p}$ is the surface of a quarter of the plastic zone.

Hence

$$
\Delta W(P Z)=4 \frac{1-N^{\prime \prime}}{1+N^{\prime \prime}} \int_{0}^{\pi / 2} \int_{0}^{r_{p}} \overline{\Delta \sigma} \cdot \overline{\Delta \varepsilon}_{p} \cdot r d r d \theta
$$

with $r_{p}$ indicates the limit of cyclic plastic zone.

If we assume that $r_{p}$ is defined by the distance for which the total equivalent strain is equal to $\overline{\Delta \varepsilon_{0}}$, we have, from (9):

$$
r_{p}=\left|\frac{\Delta K}{\Delta \sigma_{0}}\right|^{2} f_{N}(\theta)
$$

The integral (17) can be evaluated by substituting the expressions (5-8) for $\overline{\Delta \sigma}$ and $\overline{\Delta \varepsilon}_{p}$, which gives after simplification [12] :

$$
\Delta W(P Z)=2\left(1-N^{\prime}\right) \frac{1-N^{\prime \prime}}{1+N^{\prime \prime}} \Delta \sigma_{0} \cdot \Delta \varepsilon_{0}\left(\frac{\Delta K_{I}}{\Delta \sigma_{0}}\right)^{4} \int_{0}^{\pi / 2} f_{N}(\theta)^{2} d \theta
$$

This expression can be rewritten as follows:

$$
\Delta W(P Z)=\left(1-N^{\prime}\right) \frac{1-N^{\prime \prime}}{1+N^{\prime \prime}} \Delta \sigma_{0} \cdot \Delta \varepsilon_{0} \cdot S_{p z}
$$

with

$$
S_{p z}=\left[2\left(\frac{\Delta K_{I}}{\Delta \sigma_{0}}\right)^{4} \int_{0}^{\pi / 2} f_{N}(\theta)^{2} d \theta\right]
$$

indicating simply the surface of the plastic zone.

On the other hand, Engerand [24] proosed the Tresca or Von Mises criteria to compute the limit of the plastic zone. It is interesting to note that (19) enables us to express the energy dissipated throughout the plastic zone per unit thickness as a function of $\Delta K^{4}$, which conforms to the theoretical models given by Klingbeil [7], Mazari et al [15]. and Ranganathan et al [8]. Hence, in order to obtain a similar variation as a function of $\Delta K^{4}$, we propose to compute the plastic zone surface using the following expressions for the plastic rays:

$$
r_{p}(P D)=\frac{1}{2 \pi B}\left(\frac{\Delta K}{\Delta \sigma_{0}}\right)^{4} \cos ^{2} \frac{\theta}{2}\left[(1-2 v)^{2}+3 \sin ^{2} \frac{\theta}{2}\right]
$$

for plane strain, and:

$$
r_{p}(P S)=\frac{1}{2 \pi B}\left(\frac{\Delta K}{\Delta \sigma_{0}}\right)^{4} \cos ^{2} \frac{\theta}{2}\left[1+3 \sin ^{2} \frac{\theta}{2}\right]
$$


for plane stress.

By using (22) and (23), the new model proposed for the calculation of plastic energy per unit thickness can be written as follows:

$$
\Delta W(P Z)_{M}=\alpha_{M} \cdot \Delta \sigma_{0} \cdot \Delta \varepsilon_{0} \cdot S_{p z M}
$$

where $\alpha_{M}$ is a constant which depends on the material and the criterion used and $S_{M}$ indicates the surface of the plastic zone determined by expressions (22) or (23).

Hence, the total dissipated energy $\mathrm{Q}$ in the specimen is given by:

$$
Q=\Delta W(P Z) \times B
$$

\section{EXPERIMENTAL DETAILS}

\section{A. Material and specimen configurations}

The tests were conducted on two aluminum alloys, the 2024 alloy in the T351 condition and the 7075 alloy in the T7351 condition. The nominal composition and mechanical properties of these alloys are given in Tables I and II respectively. In Table II, the parameters $K$ and $n$ are computed from the relationship of Ludwik [25]:

$$
\sigma=K .\left(\varepsilon_{p}\right)^{n}
$$

TABLE I. NOMINAL COMPOSITION (IN \%) OF THE STUDIED ALLOYS.

\begin{tabular}{|c|c|c|c|c|c|c|c|c|c|}
\hline Alloy & Si & Fe & Cu & Mn & Mg & Cr & Zn & Ti & Al \\
\hline 2024 & 0.10 & 0.22 & 4.46 & 0.66 & 1.50 & 0.01 & 0.04 & 0.02 & Remain \\
\hline 7075 & 0.07 & 0.16 & 1.52 & 0.04 & 2.55 & 0.20 & 6.00 & 0.04 & Remain \\
\hline
\end{tabular}

TABLE II. NOMINAL MECHANICAL PROPERTIES OF THE STUDIED ALLOYS

\begin{tabular}{|c|c|c|}
\hline Material & 2024-T351 & 7075-T7351 \\
\hline $\begin{array}{c}\text { Conventional yield stress at 0.2\% of } \\
\text { plastic strain } \sigma_{0.2}(M P a)\end{array}$ & 318 & 470 \\
\hline Stress at fracture $\sigma_{R}(M P a)$ & 524 & 539 \\
\hline Elongation $A \%$ & 12.8 & 11.7 \\
\hline Strength coefficient $K(M P a)$ & 652 & 960.5 \\
\hline Hardening coefficient $n$ & 0.104 & 0.051 \\
\hline
\end{tabular}

The tests were carried out using compact tension specimens (CT) with thicknesses of $10 \mathrm{~mm}$ for 2024 and $6 \mathrm{~mm}$ for 7075 (Figure 4). The direction of cracking has been taken in the rolling direction. All mechanical tests were conducted using an Instron servohydraulic machine at a typical test frequency of 20 $\mathrm{Hz}$ in temperature room with a stress ratio of $R=0.5$.

$$
R=P_{\min } / P_{\max }
$$

where $P_{\min }$ and $P_{\max }$ are the minimum and maximum load in the cycle.

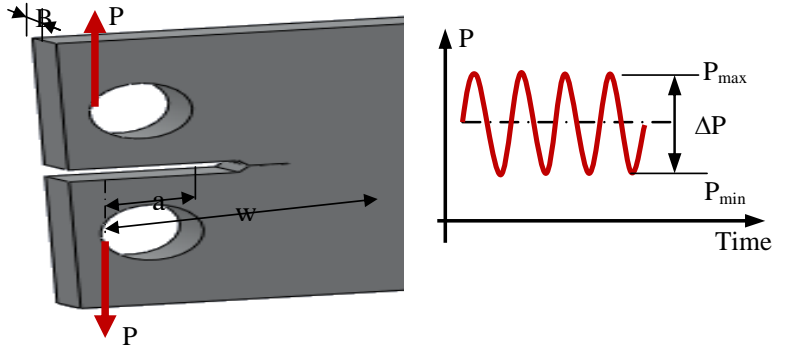

Fig. 4. Cyclic test configuration on a compact tension specimen.

The crack propagation rate was measured by optical techniques on the polished side of the specimen using a travelling microscope with a precision of $0.01 \mathrm{~mm}$. The total dissipated energy was evaluated by the area locked up inside the recorded hysteresis cycles. The stress intensity range for this geometry is given by Newman [26]:

$$
\Delta K=\frac{\Delta P}{B \cdot \sqrt{w}} f(z) \text { with } z=\frac{a}{w}
$$

where $w$ and $B$ are respectively the width and the thickness of the specimen, a, is the crack length, and $\Delta P=P_{\max }-P_{\min }$, is the amplitude applied load. In order to obtain more precision, two functions of compliance $f(z)$ were used:

- $\quad$ for $0.2 \prec z \prec 0.3[26]$ :

$$
\begin{aligned}
& f(z)=4.55-40.32 z+414.7 z^{2}-1698 z^{3}+3781 z^{4} \\
& -4287 z^{5}+2017 z^{6}
\end{aligned}
$$

- for $0.3 \prec z \prec 0.7 \quad$ [27]:

$$
\begin{aligned}
f(z)= & 29.6 z^{0.5}-185.5 z^{1.5}+655.7 z^{2.5} \\
& -1017 z^{3.5}+638.9 z^{4.5}
\end{aligned}
$$

B. Identification of the cyclic plastic strain energy parameters

The results obtained after the dentification of the cyclic plastic strain energy parameters are summarized in Table III.

TABLE III. CYCLIC PLASTIC STRAIN ENERGY PARAMETERS

\begin{tabular}{|c|c|c|c|c|c|c|}
\hline \multicolumn{7}{|c|}{ For 2024-T351 } \\
\hline$\Delta \sigma_{0}$ & $\Delta \varepsilon_{0}$ & $\alpha_{M}$ & $N^{\prime}$ & $N^{\prime \prime}$ & Integral & $\Delta W / \Delta K^{4}$ \\
\hline 914 & 0.0111 & $6.67 \mathrm{E}-4$ & 0.148 & 0.078 & 0.0138 & $2.92 \mathrm{E}-13$ \\
\hline \multicolumn{7}{|c|}{ For 7075-T7351 } \\
\hline$\Delta \sigma 0$ & $\Delta \varepsilon 0$ & $\alpha_{M}$ & $N^{\prime}$ & $N^{\prime \prime}$ & Integral & $\Delta W / \Delta K^{4}$ \\
\hline 705 & 0.00849 & $1.26 \mathrm{E}-5$ & 0.166 & 0.0765 & 0.0140 & $4.85 \mathrm{E}-13$ \\
\hline
\end{tabular}

For the particular materials under study, we finally obtain:

- $\quad$ For 2024-T351:

$$
\Delta W=2,92.10^{-13} \Delta K^{4} \text { Joule } / \mathrm{m}
$$

- $\quad$ For 7075-T7351: 


$$
\Delta W=4,85.10^{-13} \Delta K^{4} \text { Joule } / \mathrm{m}
$$

\section{Identification of Paris law}

Fatigue crack growth in the CT specimens was modeled according to the Paris Law, where the FCG rate, $d a / d N$, is described in terms of the stress intensity range $\Delta K$ according to the following relationship [28]:

$$
\frac{d a}{d N}=C(\Delta K)^{m}
$$

where $C$ and $m$ are the fatigue crack growth coefficient and exponent, respectively.

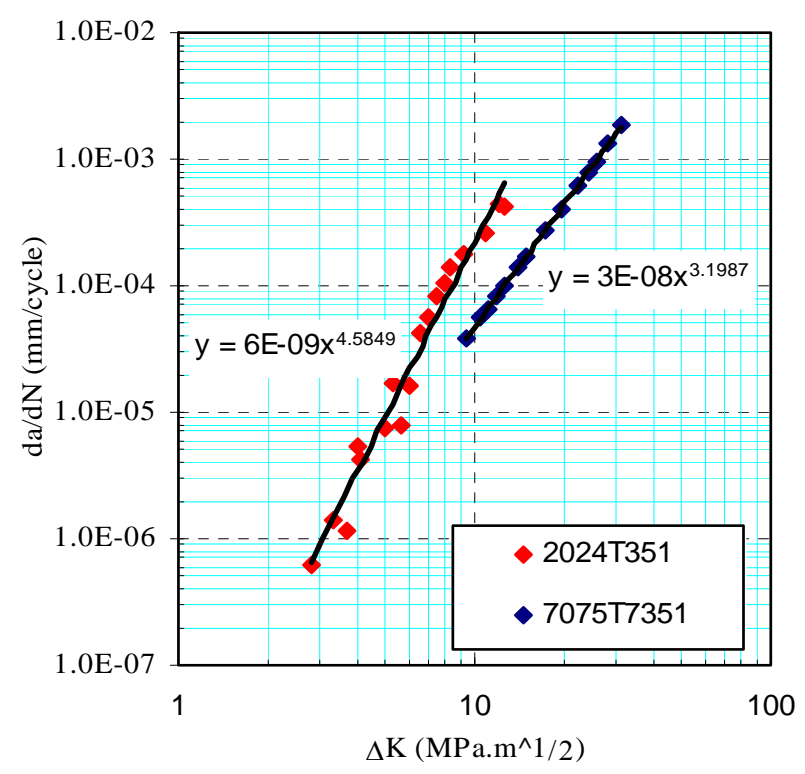

Fig. 5. Fitting of experimental data with a Paris law

Figure 5 shows typical results of the experiments used in constructing plots of $\log (d a / d N)$ in terms of $\log (\Delta K)$ for each aluminum alloy. A power law model was fit to the steady state region (stage II) of fatigue crack growth and the Paris Law coefficient $(C)$ and exponent $(m)$ were determined for each specimen that underwent stable fatigue crack growth. A logarithmic scale was used to represent the curve as a straight line. Thus, a linear regression returns the material parameters $C$ and $m$. By identifying the Paris law parameters according to experimental data's of the two alloys, we found that $C=6.0 \mathrm{E}-9$ and $m=4.5849$ with a slope of 4.58 for 2024-T351. However, for 7075-T7351: $C=3.0 \mathrm{E}-8$ and $m=3.1987$ with a slope of 3.20.

It is worth noting that the 2024 aluminum alloy remains as an important aircraft structural material due to its extremely good damage tolerance and high resistance to fatigue crack propagation [29]. However, the 7075-T7351 offers good stresscorrosion cracking resistance [30].

\section{IMPLEMENTATION OF THE PROPOSED APPROACH}

A computer program has been written in Matlab language. The program was developed to calculate the stress intensity factor, the size of the plastic zone, the crack growth rate, the number of cycles and the cyclic plastic strain energy. Conditions of plane strain and Von Mises criterion were considered. First, we specify CT specimen dimensions ( $B$ : thickness, $w$ : width, $a_{0}$ : initial crack length, $a_{E n d}$ : crack length for forced termination), quantities relevant to material properties ( $E$ : Young's modulus, $v$ : Poisson's ratio, $\sigma_{y}$ : yield stress, $C$ and $m$ : material constants of Paris law and the amplitude of the applied load). Then, the program computes the stress intensity factor range $\Delta K$, the plastic radius $r_{p}$, the fatigue crack growth rate $d a / d N$, and the energetic parameters as shown by the flaw-chart in Figure 6 .

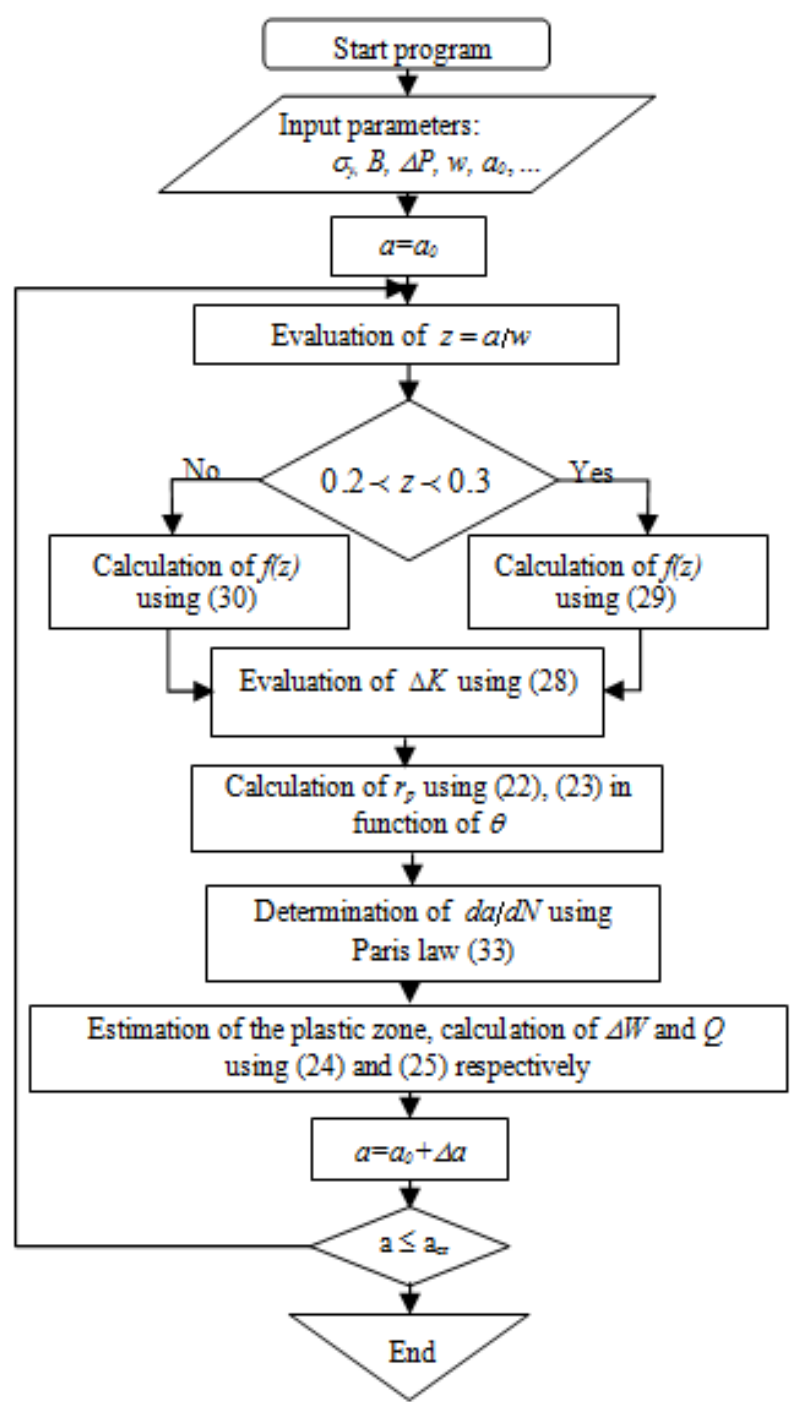

Fig. 6. Flow chart of the numerical process. 


\section{RESULTS AND DISCUSSION}

It is worth noting that the evolution of the cyclic plastic strain energy is directly related to the change of the plastic zone size at the crack tip as described in section (II.B). In what follows, we will first deal with the evolution of the plastic zone. Then, we will analyze in detail the evolution of the cyclic plastic strain energy and the speed of crack propagation.

\section{A. Evolution of the plastic zone}

The purpose of this section is to study in detail the evolution of the plastic zone at the crack tip during the crack growth on the two aluminum alloys. It should be noted that, at the crack tip of a ductile material, the strain fields are significant and leading to a considerable extent of the plastic zone. In this case, the mechanical energy at the crack tip is absorbed by the material in the form of linear defects (dislocations) [20,31].On the other hand, the size of the plastic zone depends not only on the nature of the materials, but also on the intensity of the mechanical energy at the crack tip, the geometry and the size of the crack. Noting that the hardening (maximum consolidation of material) characterized by its rate, can significantly slow the extension of the plastic zone.

Figure 7 shows the evolution of the plastic zone for both aluminum alloys (2024 and 7075) using Tracey model (Figure $7 \mathrm{a}$ ) and the proposed model (Figure 7b). It is found that the size of the plastic zone calculated by the proposed model is about 16 times (for 2024) and 18 times (for 7075) greater than that calculated by the Tracey model. Furthermore, we observe that the 2024 alloy, whose mechanical properties of ductility are higher than 7075, presents the largest size of the plastic zone.

We have obtained for:

- $\quad$ Tracey model: $\quad \mathrm{S}(\mathrm{PZ})$ of $2024=2,37 \times \mathrm{S}(\mathrm{PZ})$ of 7075.

- $\quad$ Proposed model: S(PZ) of $2024=2,12 \times \mathrm{S}(\mathrm{PZ})$ of 7075.

\section{B. Evolution of the total dissipated energy in the specimen}

It should be noted that the crack extension which leads to the fracture occurs when the provided energy is sufficient to overcome the material strength. Assuming that this energy is mainly dissipated in the plastic zone, a comparison can be made between the measured values and those predicted theoretically as shown in Figure 8 by assuming propagation in mode I.

\section{Evolution of the total dissipated energy in the specimen}

It should be noted that the crack extension which leads to the fracture occurs when the provided energy is sufficient to overcome the material strength. Assuming that this energy is mainly dissipated in the plastic zone, a comparison can be made between the measured values and those predicted theoretically as shown in Figure 8 by assuming propagation in mode I.

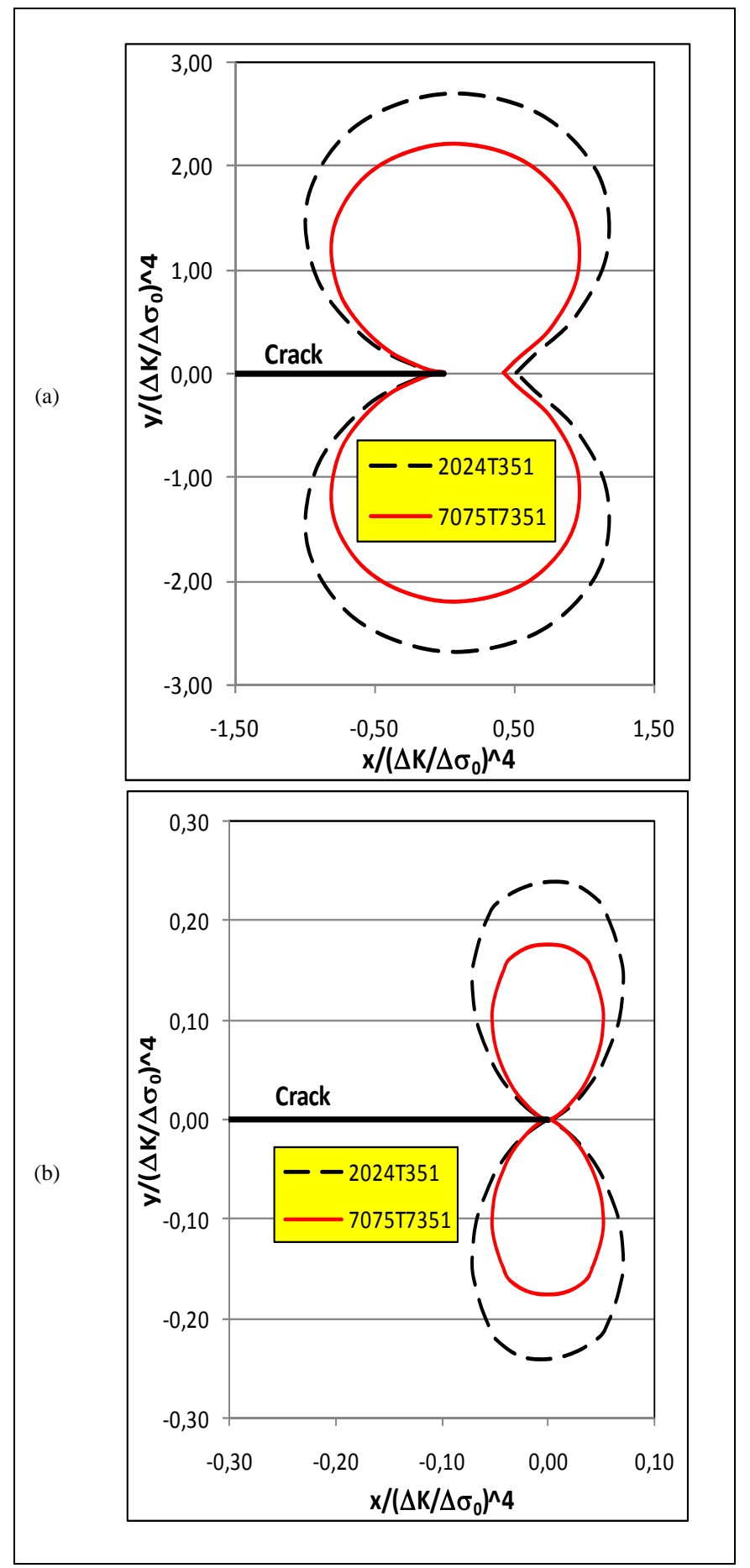

Fig. 7. Evolution of the normalized plastic zone in the case of : (a) the Tracey model and (b) the proposed model for both aluminum alloys.

Figure 8 illustrates the evolution of the total dissipated energy according to the amplitude of the stress intensity factor $\Delta K$ for both types of aluminum alloys in the case of a constant stress ratio of $\mathrm{R}=0.5$. It can be seen that the theoretical estimates given by the Tracey model are much lower than the experimental measurements. However, a good prediction has been obtained by the proposed model. Noting that for 2024 
(Figure 8a), $Q$ calculated by the Tracey model is about 11 to 76 times weaker than the measured $Q$; the difference is less for high values of $\Delta K$. The same trends have been also found for 7075 (Figure 8b). The gap between the theoretical estimates of Tracey and the measured values ranges from 3 , for high values of $\Delta K$, to 10 for the low values of $\Delta K$. This difference can be allotted in a great part to the size of the estimated plastic zone which is much lower than that measured especially for materials that exhibit a high ductility as that of 2024-T351 in comparison with that of 7075-T7531. On the other hand, it may be noted from Figure 8, that the results obtained by the proposed model are in good agreement with experimental data for both types of aluminum alloys. It is also observed that for the same value of $\Delta K$, the total dissipated energy of 2024-T351 is higher than that of 7075-T7531.

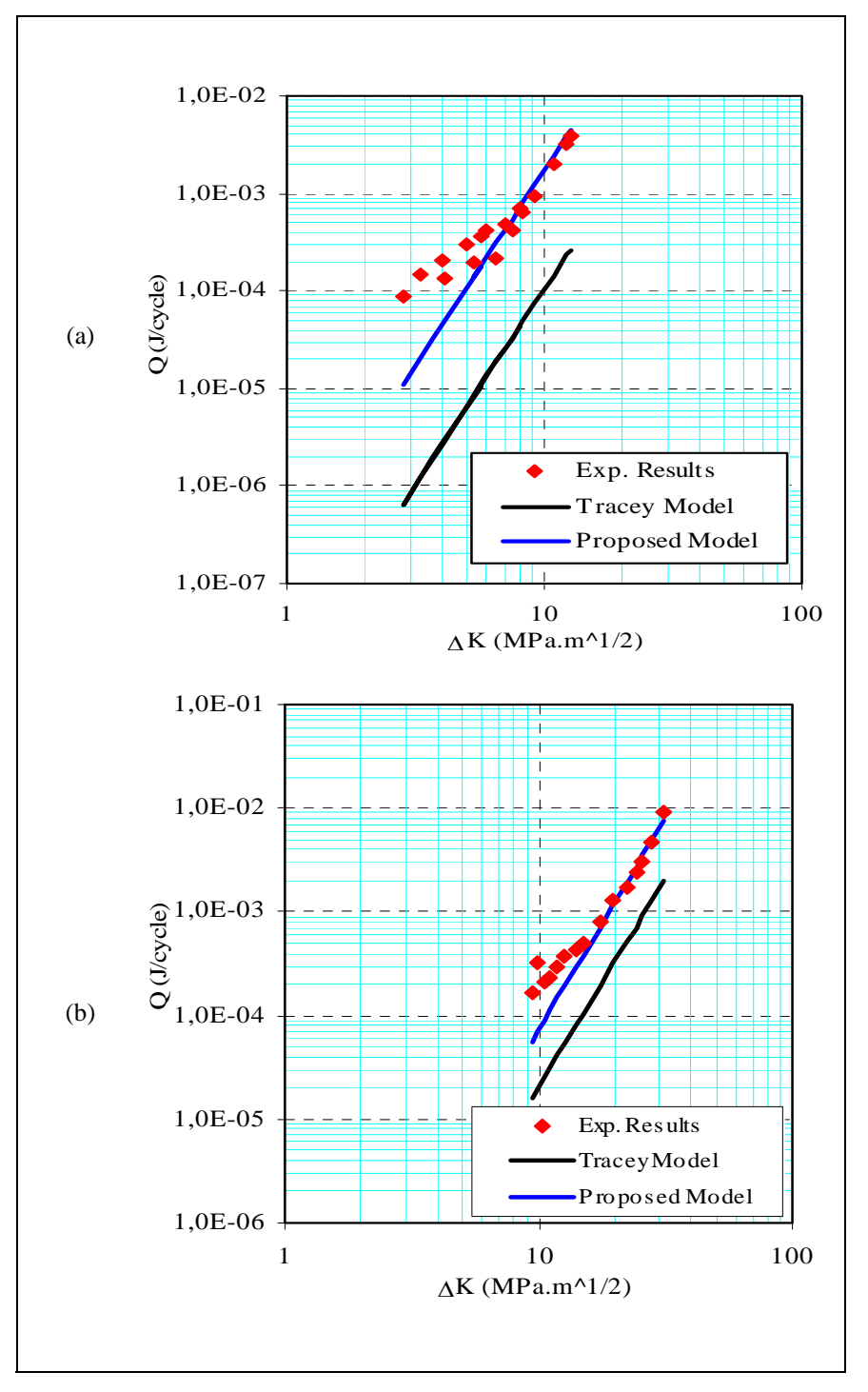

Fig. 8. Comparison of measured and estimated dissipated energy per cycle for (a) 2024-T351 and (b) 7075-T7351.

\section{RELATIONSHIP BETWEEN $Q$ AND $d a / d N$}

The evolution of the crack growth rate is studied as a function of energetic parameters in order to interpret the crack behavior in various elucidated regimes. Figure 9 shows the evolution of $d a / d N$ in terms of the total dissipated energy $Q$ for $\mathrm{R}=0.5$. For both aluminum alloys, the experimental results can be subdivided into two distinct stages as shown in Figure 9. In the case of 2024 , the stage $\mathrm{I}$ is defined by $d a / d N \leq 2 \times 10^{-5}$ $\mathrm{mm} /$ cycle.

In this stage we note a strong decrease in crack propagation speed with the total dissipated energy.

A logarithmic approximation can be used in this stage. The relationship obtained is given by:

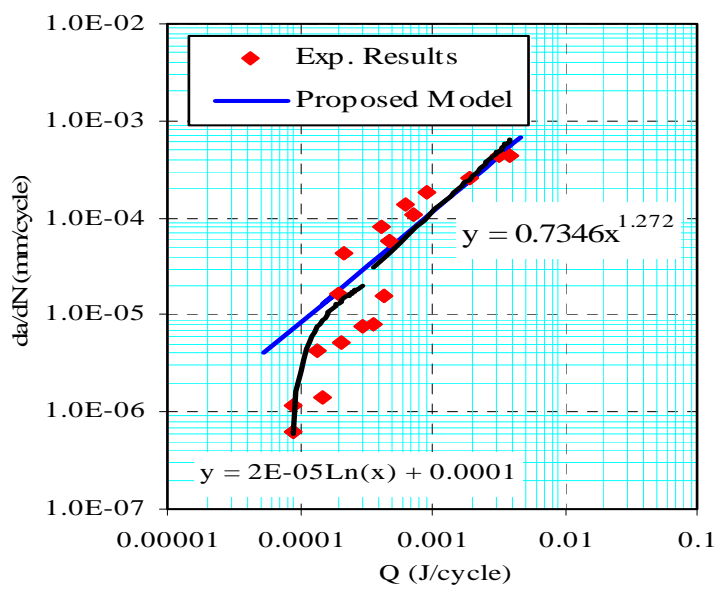

Fig. 9. Comparison of measured Evolution of $d a / d N$ with $Q$ for 2024T351

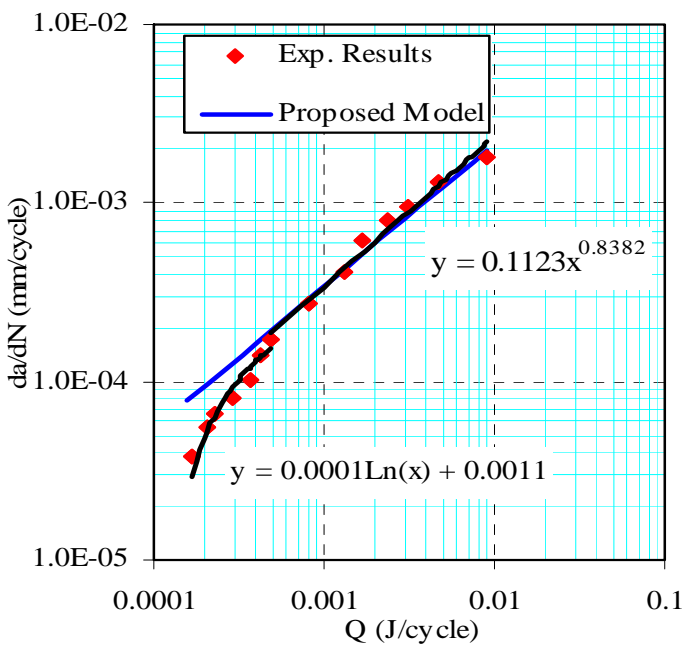

Fig. 10. Evolution of $d a / d N$ with $Q$ for 7075-T7351. The black lines represent the regression curves of experimental results using power and logarithmic functions 
Stage II is defined by $d a / d N \geq 2 \times 10^{-5} \mathrm{~mm} /$ cycle. This region exhibits a stable evolution of the crack growth and a power law can be used as an approximation in this stage:

$$
\frac{d a}{d N}=0.7346 Q^{1.272}
$$

In the case of 7075 , stage $\mathrm{I}$ is defined by $d a / d N \leq 2.10^{-4}$ $\mathrm{mm} /$ cycle. The evolution of the crack growth in this regime is unstable. If we use a logarithmic function, we can determine a relationship of the form:

$$
\frac{d a}{d N}=0.0001 \operatorname{Ln}(Q)+0.0011
$$

Stage II is defined by $\mathrm{da} / \mathrm{dN} \geq 2 \cdot 10^{-4} \mathrm{~mm} /$ cycle. The obtained law has the following form:

$$
\frac{d a}{d N}=0.1123 Q^{0.8382}
$$

One can observe that there is a good agreement in the stage II between experimental data and the results obtained by the proposed model for both types of aluminum alloys. Furthermore, the straight lines of slope 0.316 for 2024 and 0.086 for 7075 can be deduced from the proposed model. Indeed, we obtain the following expressions:

$$
\begin{array}{ll}
\frac{d a}{d N}=0.316 Q^{1.1462} & \text { for 2024T351 } \\
\frac{d a}{d N}=0.086 Q^{0.7997} & \text { for 7075T7531 }
\end{array}
$$

However a significant difference was found in Stage I between experimental data and the results of the proposed model. This limits, therefore, the application of the proposed model in the stage where the crack propagation is stable.

\section{CONCLUSIONS}

In this study an energy based approach of fatigue crack growth has been proposed. This approach provides a direct link between the cyclic plastic strain energy and the plastic zone at the crack tip. In order to validate the model, experimental data conducted on CT specimens of aluminum alloys (2024-T351 and 7075-T7351), under constant amplitude with a stress ratio of $R=0.5$ and mode I loading, have been exploited. The following conclusions have been drawn:

- $\quad$ The measured values of the cyclic plastic strain energy for both types of aluminum alloys are substantially higher than those calculated by the Tracey model. However, a relatively good agreement has been found between the experimental data and the results obtained by the proposed model.

- A correct modeling of the plastic zone is necessary to accurately determine the total cyclic plastic dissipation at the crack tip. The size of the plastic zone calculated by the proposed model is about 16 to 18 times greater than that calculated by the Tracey model.
Finally, it should be noted that the simplicity of the current modeling approach limits its ability to account for the crack closure, the environment and the variable amplitude loadings effects, which are topics of ongoing research.

\section{ACKNOWLEDGEMENTS}

It should be noted that the experimental tests were carried out and provided within the framework of a cooperation project "CMEP $389 \mathrm{MDU}$ 97" between the Laboratory of Mechanics and Energetic (University Djillali Liabes of Sidi Bel Abbes (Algeria)) and the Laboratory of Mechanics and Rheology (University François Rabelais of Tours (France)). The authors especially thank Professor N. Ranganathan and his team for their support and assistance.

\section{REFERENCES}

[1] J. R. Rice, “The mechanics of crack tip deformation and extension by fatigue”, Fatigue Crack Propagation Special Technical Publication 415, ASTM, pp. 247-311, Philadelphia, 1967

[2] I. Shozo, I. Yoshito, M. E. Fine, "Plastic work during fatigue crack propagation in a high strength low alloy steel and in 7050 AL-Alloy”, Engineering Fracture Mechanics, Vol. 9, No. 1, pp. 123-136, 1977

[3] P. K. Liaw, S. I. Kwun, M. E. Fine, "Plastic work of fatigue crack propagation in steels and aluminum alloys”, Metallurgical Transactions A, Vol. 12, No. 1, pp. 49-55, 1981

[4] C. Saix, P. Jouanna, "Analyse de la dissipation plastique dans des pièces métalliques minces”, Journal de Mécanique Appliquée, Vol. 5, No. 1, pp. 65-93, 1981

[5] S. R. Bodner, D. L. Davidson, J. Lankford "A description of fatigue crack growth in terms of plastic work”, Engineering Fracture Mechanics, Vol. 17. No. 2, pp. 189-191, 1983

[6] A. D. Joseph, T. S. Gross, "Comparison of techniques for the measurement of plastic work of fatigue crack growth in low carbon steel”, Engineering Fracture Mechanics, Vol. 21, No. 1, pp. 63-74, 1985

[7] N. W. Klingbeil, "A total dissipated energy theory of fatigue crack growth in ductile solids", International Journal of Fatigue, Vol. 25, No. 2, pp. 117-128, 2003

[8] N. Ranganathan, F. Chalon, S. Meo, "Some aspects of the energy based approach to fatigue crack propagation”, International Journal of Fatigue, Vol. 30, No. 10-11, pp. 1921-1929, 2008

[9] R. Jones, M. Krishnapillai, K. Cairns, N. Matthews, “Application of infrared thermography to study crack growth and fatigue life extension procedures”, Fatigue \& Fracture of Engineering Materials \& Structures, Vol. 33, No. 12, pp. 871-884, 2010

[10] J. S. Daily, N. W. Klingbeil, "Plastic dissipation energy at a bimaterial crack tip under cyclic loading”, International Journal of Fatigue, Vol. 32, No. 10 , pp. $1710-1723,2010$

[11] J. Weertman, "Theory of fatigue crack growth based on a BCS crack theory with work hardening”, International Journal of Fracture.; Vol. 9, No. 2. 125-131, 1973

[12] M .Mazari, “Contribution à l'étude d’une approche énergétique de la propagation des fissures de fatigue”, Thèse de doctorat, Université de Sidi Bel Abbès, Algérie, 2003

[13] J. Morrow, "Cyclic Plastic Strain Energy and Fatigue of Metals” in Internal friction, damping, and cyclic plasticity, ASTM STP 378, American Society for Testing and Material, 1965

[14] J. S. Daily, N. W. Klingbeil. "Plastic dissipation in fatigue crack growth under mixed mode loading", International Journal of Fatigue, Vol. 26, No. 7, pp.727-738, 2004

[15] M. Mazari, B. Bouchouicha, M. Zemri, M. Benguediab, N. Ranganathan, "Fatigue crack propagation analyses based on plastic energy approach”, Computational Materials Science, Vol. 41, No. 3, pp. 344-349, 2008

[16] N. Ranganathan, “Contribution au développement d'une approche énergétique à la propagation d'une fissure de fatigue”, Thèse de doctorat, Université de Poitiers. France, 1985 
[17] W. Wang, H. Hsu, "Fatigue crack growth rate of metal by plastic energy damage accumulation theory", Journal of Engineering Mechanics, Vol. 120, No. 4, pp. 776-795, 1994

[18] S. M. Beden, S. Abdullah, A. K. Ariffin. "Review of Fatigue Crack Propagation Models for Metallic Components”. European Journal of Scientific Research, Vol. 28. No. 3, pp. 364-397, 2009

[19] R. O. Ritchie, "Mechanisms of fatigue-crack propagation in ductile and brittle solids”. International Journal of Fracture., Vol. 100, No. 1, pp. 5583, 1999

[20] N. Ranganathan, K. Jendoubi, M. Benguediab, J. Petit, "Effect of R ratio and $\Delta \mathrm{K}$ level on the hysteretic energy dissipated during fatigue crack propagation”, Scripta Metallurgica, Vol. 21, No. 8, pp. 1045-1049, 1987

[21] D. M. Tracey, "Finite element solution for crack-tip behavior in smallscale yielding”, Journal of Engineering Materials and Technology, Vol. 98, No. 2, pp. 146-151, 1976

[22] G. Chalant, L. Remy, "Plastic strain distribution at the tip of a fatigue crack. Application to fatigue crack closure in the threshold regime", Engineering Fracture Mechanics, Vol. 16, No. 5, pp. 707-720, 1982

[23] G. Chalant, "Fissuration par fatigue d'alliages cobalt-Nickel : Discussion d'un modèle mécanique de propagation”. Thèse de doctorat de l'Ecole des Mines de Paris, 1981

[24] J. L. Engerand, Mécanique de la rupture, Ed. Techniques Ingénieur, 1990

[25] P. Ludwik, Elemente der Technologischen Mechanik, Springer-Verlag OHG, Berlin, 1909

[26] J. C. Newman, "Stress analysis of the compact specimen including the effects of pin loading fracture analysis”, ASTM STP 560, pp. 105-121, 1974

[27] J. E. Srawley, B. Gross, "Stress intensity factors for bend and compact specimens”, Engineering Fracture Mechanics, Vol. 4, No. 3, pp. 587589, 1972

[28] P. C. Paris, F. A. Erdogan, "A critical analysis of crack propagation laws”, Journal of Basic Engineering, Vol. 85, No. 4, pp. 528-533, 1963

[29] D. Altenpohl, Aluminium: Technology, application and environment. A profile of a modern metal: Aluminum from Within, 6th Edition, Wiley, 2010

[30] P. S. Pao, S. J. Gill , C. R. Feng, “On fatigue crack initiation from corrosion pits in 7075-T7351 aluminum alloy”, Scripta Materialia, Vol. 43, No. 5, pp. 391-396, 2000

[31] J. W. Kysar, "Energy dissipation mechanisms in ductile fracture", Journal of the Mechanics and Physics of Solids, Vol. 51, No. 5, pp. 795824, 2003 